\title{
Unilateral Eye Findings: A Rare Herald of Acute Leukemia
}

\author{
Avni V. Patel ${ }^{a} \quad J o h n$ B. Miller ${ }^{a, b}$ Rajneesh Nath ${ }^{c}$ Helen A. Shih ${ }^{d}$ \\ Michael K. Yoon ${ }^{\mathrm{a}, \mathrm{e}}$ Suzanne K. Freitag ${ }^{\mathrm{a}, \mathrm{e}}$ George Papaliodis ${ }^{\mathrm{a}} \mathrm{f}$ \\ Teresa C. Chen ${ }^{a, g}$ Dean Eliott ${ }^{a, b}$ Ivana K. Kimª, \\ ${ }^{a}$ Department of Ophthalmology, Harvard Medical School, and ${ }^{b}$ Retina Service, Massachusetts Eye and Ear \\ Infirmary, Boston, Mass., 'Department of Hematology/Oncology, UMass Memorial Medical Center and University

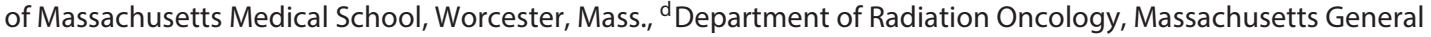 \\ Hospital and Harvard Medical School, e Department of Ophthalmic Plastic Surgery, ${ }^{f}$ Ocular Immunology and Uveitis \\ Service, and ${ }^{9}$ Glaucoma Service, Massachusetts Eye and Ear Infirmary, Boston, Mass., USA
}

\section{Key Words}

Choroidal infiltration - Leukemia - Ocular manifestations of leukemia $\cdot$ Ocular oncology $\cdot$ Radiation

\begin{abstract}
Background/Aim: Unilateral choroidal infiltration as the initial manifestation of leukemic relapse in adults is rare, particularly after an extended period of remission. This report describes this unique ophthalmic presentation, highlights the associated diagnostic challenges, and reviews the literature. Methods: Two cases are described and an extensive literature review was conducted. Results: A 59-year-old male with acute lymphoid leukemia, in remission for 18 months, presented with unilateral scleritis, exudative retinal detachment, and choroidal thickening. A 57-year-old male with a history of acute myeloid leukemia, in remission for 4 years, presented with unilateral choroidal thickening leading to secondary angle closure. In both cases, there was a significant lag from the onset of eye symptoms to establishing a systemic diagnosis of acute leukemia, leading to a delay in definitive systemic treatment, despite a high suspicion of disease based on ophthalmic findings. Conclusions: These
\end{abstract}

\section{KARGER}

(c) 2016 S. Karger AG, Base

2296-4681/16/0023-0166\$39.50/0

E-Mail karger@karger.com

www.karger.com/oop two cases illustrate the fundus findings consistent with leukemic choroidal infiltration that can represent the first sign of relapsed leukemia. The successful treatment of these patients hinges on collaboration between ophthalmologists and oncologists to optimize patient outcomes, highlighting the need for both groups to be aware of this rare ophthalmic presentation.

C 2016 S. Karger AG, Basel

\section{Introduction}

While ophthalmic involvement is a well-documented complication of leukemia, it is rare for ophthalmic findings to be the initial manifestation of new or relapsed disease $[1,2]$. Additionally, when eye findings are present, they usually manifest simultaneously and bilaterally in systemic diseases such as leukemia. We report two cases of unilateral ocular involvement as the initial manifestation of relapsed leukemia in two patients without prior evidence of central nervous system involvement. Additionally, a thorough literature search was conducted (PubMed, keywords: 'leukemia', 'ocular', 'eye', and 

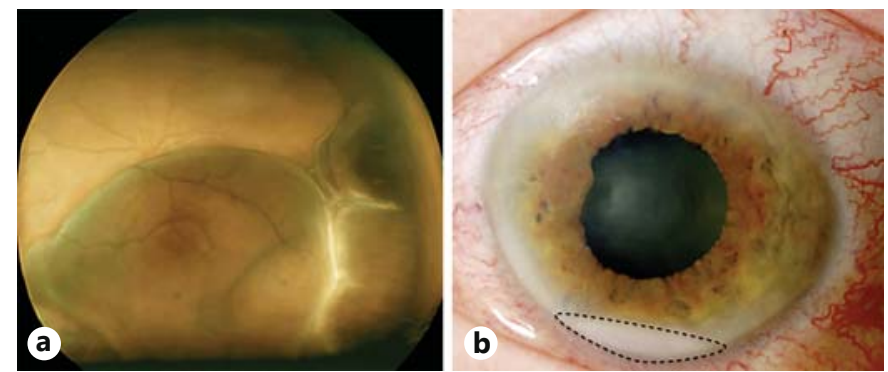

Fig. 1. a Fundus photo of the patient's left eye (case 1) shows an inferior serous retinal detachment with choroidal thickening and effusions. b Anterior segment photo of the patient's left eye (case 1) demonstrating the blood-tinged leukemic hypopyon with the dashed line outlining hypopyon.

'adult') to review the variety of presentations of ocular leukemic disease and highlight the uncommon features of these cases. These examples emphasize the need for ophthalmologists and oncologists alike to be aware of the full spectrum of ocular signs of leukemia, as a prompt and thorough systemic workup and treatment in these cases may not only salvage the patient's sight but also be lifesaving.

\section{Case 1}

A 59-year-old male physician with Philadelphia chromosome (BCR-ABL)-positive acute lymphoid leukemia (ALL), in complete remission after myeloablative chemotherapy and matched related donor hematopoietic stem cell transplant 18 months prior, presented with progressive irritation, redness, and decreased vision in his left eye over 1 month.

His past ocular history was notable for recent episodes of anterior uveitis and scleritis in the left eye only, which were previously treated with topical and systemic steroids, difluprednate and prednisone, respectively. He was also on imatinib mesylate (Gleevec, Novartis Pharmaceutical Corp., East Hanover, N.J., USA) as maintenance therapy for his hematologic disease.

On presentation to our clinic, his best-corrected visual acuity was 20/20 in the right eye (OD) and counting fingers in the left eye (OS). His intraocular pressures were $24 \mathrm{~mm} \mathrm{Hg}$ OD and $20 \mathrm{~mm}$ Hg OS. The slit-lamp examination of the right eye was unremarkable. The left eye had rare cells in the anterior chamber, mild nuclear sclerotic cataract, and $2+$ vitreous cells. The fundus exam was notable for a retinal detachment with shifting subretinal fluid, with inferior choroidal thickening and effusions (fig. 1a). Central serous retinopathy was an initial consideration for this patient's presentation, but most concerning to the ophthalmologist was the potential diagnosis of leukemic infiltration causing the choroidal thickening and serous detachment given the patient's past medical history. However, the patient's bone marrow biopsy 3 months prior did not show any signs of recurrence, and molecular studies showed that BCR-ABL or the Philadelphia chromosome, a fusion gene of chro-

Unilateral Eye Findings: A Rare Herald of Acute Leukemia mosomes 9 and 22 present in this patient's form of ALL, was not detected. Given this recent negative testing, these eye findings alone were not accepted as signs of relapsed ALL by his oncologist.

One month later, the patient returned with worsening scleritis, uveitis, and a blood-tinged hypopyon with neovascularization of the iris (fig. 1b). At this time, he had a total retinal detachment with persistent choroidal effusions further increasing the ophthalmologist's suspicion for leukemic recurrence. However, the patient's oncologists remained uncertain that the ocular manifestations could be a sign of relapsed disease given the lack of systemic findings. He was followed with repeat BCR-ABL testing every few months, which remained negative. Over the next 6 months, the patient's vision continued to deteriorate to light perception, and the possibility of malignant infiltration was discussed repeatedly; however, the patient deferred biopsy or surgical intervention for his ocular manifestations. When the eye became painful, enucleation of the eye was considered; however, prior to the procedure, he presented to the emergency room with severe headache, fatigue, and weight loss. Cerebrospinal fluid sampling at that time showed clear evidence of central nervous system leukemia, with over 3,000 white cells, $72 \%$ of which were blasts. At this time, he received intrathecal chemotherapy and high-dose methotrexate. Systemic therapy with dasatinib (Sprycel, Bristol-Meyers Squibb, Princeton, N.J., USA) was also initiated, and radiation treatment of $20 \mathrm{~Gy}$ to the left orbit was delivered. His vision remained 20/25 in the right eye without any fundus changes and light perception in the left eye without pain. He is currently receiving ongoing neuro-oncologic treatment for his relapsed disease.

\section{Case 2}

A 57-year-old male with a history of acute myeloid leukemia (AML; normal cytogenetics with Flt-3 and NPM-negative), in complete remission for 4 years after induction chemotherapy with daunorubicin and high-dose cytarabine (HIDAC), consolidation chemotherapy with 3 cycles of HIDAC and autologous stem cell transplant followed by maintenance 5 -azacytadine, was admitted to an outside hospital with progressive right eye pain, redness, and swelling. He was presumed to have a right orbital cellulitis, but a lack of improvement on broad-spectrum antimicrobials prompted transfer to our institution.

On presentation, the patient's visual acuity was decreased to 20/200 OD and stable at 20/20 OS. His intraocular pressure was elevated at $49 \mathrm{~mm} \mathrm{Hg} \mathrm{OD}$ and normal at $17 \mathrm{~mm} \mathrm{Hg}$ OS. Slit-lamp examination was notable for right periorbital erythema and edema, diffusely injected conjunctiva, microcystic corneal edema, and a shallow anterior chamber. Examination of the left eye was unremarkable, including a deep and quiet anterior chamber. Gonioscopy revealed no visible angle structures OD, consistent with a closed angle, while OS, the angle was open to scleral spur $360^{\circ}$.

A B-scan ultrasound showed a diffusely thickened choroid, and ultrasound biomicroscopy confirmed secondary angle closure due to anterior rotation of the lens-iris diaphragm. Intraocular pressure-lowering medications and cyclopentolate were started. After the intraocular pressure began to improve, diffuse irregular choroidal elevation was seen on fundus examination of the right eye. Fluorescein angiography showed pinpoint leakage in the inferotemporal periphery and diffuse late subretinal leakage (fig. 2). 

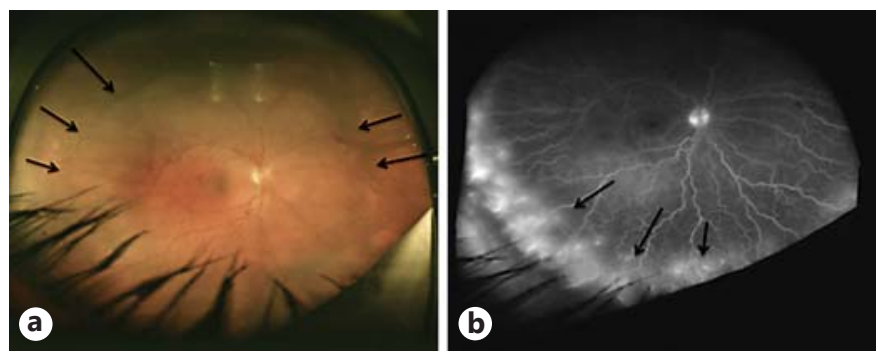

Fig. 2. a Fundus photo of the patient's right eye (case 2) showing diffuse, irregular choroidal elevation and rare intraretinal hemorrhages. Arrows help demarcate areas where prominent elevation is seen. b Fluorescein angiography (case 2) demonstrating pinpoint leakage in the inferotemporal periphery, shown with arrows.
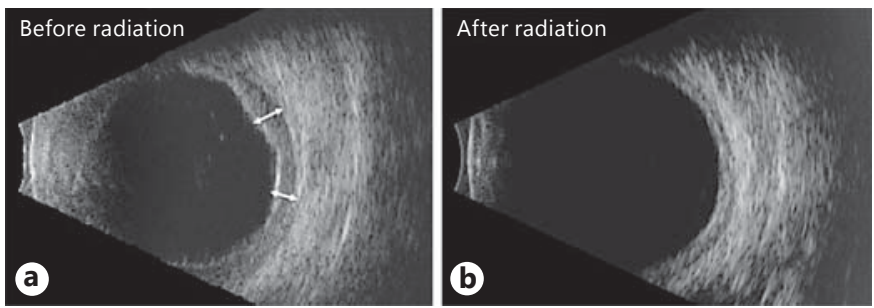

Fig. 3. B-scan ultrasonography images of the patient's right eye (case 2) before (a) and after radiation (b) with resolution of the diffuse choroidal thickening with radiation treatment. Arrows delineate thickening in the ultrasound image before radiation.

mon presentation of new or relapsed leukemic disease, but it has been previously reported in cases of ALL and AML in the adult population. In the majority of reported cases of serous retinal detachment or choroidal infiltration, the systemic disease had been diagnosed prior to the development of ocular symptoms [3-9]. A review of the literature revealed only 5 cases of decreased vision and serous retinal detachment as initial presenting signs of new leukemia in an adult patient [10-14]. Kim et al. [10] and Yoshida et al. [11] both described adult women presenting with bilateral serous retinal detachments as the initial sign of Philadelphia chromosome-positive ALL. In both cases, the serous detachments completely resolved with full recovery of vision once systemic chemotherapy was promptly initiated. Riss et al. [12] and Caillaux et al. [13] described similar presentations of bilateral serous detachments revealing a new diagnosis of AML in two adult patients. In all of these cases, the patients presented with bilateral simultaneous serous retinal detachments while in both of our patients, the initial ophthalmic involvement was unilateral.

There are additionally four cases in the literature of retinal involvement in adults as the initial sign of relapsed leukemic disease as in our patients. However, all prior cases had a much shorter time from remission to retinal infiltrative disease as a marker of relapse, with only 3-5 months of a disease-free period compared to the 18 months and 4 years seen in each of our patients [14-17]. Wu et al. [15] described a 30-year-old woman with AML type M4-M5 who was in complete remission documented by bone marrow aspiration and presented with decreased vision, bilateral serous retinal detachments, and choroidal infiltration 3 months later. As she had no systemic evidence of relapsed disease, oncology initially de- 
ferred treatment. Only after choroidal biopsy and repeat bone marrow workup 3 months later demonstrated relapsed disease was chemotherapy restarted [15]. Similarly, three other cases are reported in the literature, one with bilateral choroidal elevation and two with bilateral simultaneous serous retinal detachments. The time from remission to presentation with ocular symptoms ranged from just a few weeks to 5 months after remission in these cases $[14,16,17]$.

Further, choroidal infiltration causing angle closure, as in our second case, is exceedingly rare. There is only one other report of a patient who presented with bilateral angle closure and proptosis due to uveoscleral thickening in the setting of leukemia [18]. This patient already had an established diagnosis of AML at the time of visual symptoms, and his presentation was bilateral. In the case of our patient, the disease was in remission for several years, and he did not develop symptoms in the second eye until after radiation treatment was initiated for the first eye. In both cases presented in this report, the unilaterality of symptoms and findings made the diagnosis of relapsed disease particularly challenging.

Ocular manifestations of leukemia may present as direct infiltration of the conjunctiva, uvea, optic nerve or orbit or as secondary changes due to the hematologic changes associated with leukemia such as anemia, thrombocytopenia, hyperviscosity, and opportunistic infections $[2,19]$. Studies have shown varying prevalence of leukemic involvement of the eye ranging from $50-80 \%$ with a higher incidence of involvement in histopathologic postmortem studies $[1,20]$. This is likely explained by the often subtle clinical signs that may indicate ocular involvement, particularly choroidal involvement. A higher incidence of ocular involvement, including choroidal infiltration, is seen in eyes with acute leukemia compared to chronic leukemia: 80 versus $29 \%$ [20]. If clinically evident choroidal infiltration is present, it most often presents with retinal pigmentary changes, thickening, and serous retinal detachment in the most conspicuous cases [1]. Serous retinal detachment is thought to occur due to direct leukemic infiltration of the choroid causing decreased blood flow in the choriocapillaris, resulting in ischemia to the overlying retinal pigment epithelium (RPE) and disrupting intercellular tight junctions and the ability of the RPE to effectively pump fluid $[21,22]$. Special ancillary tests, such as fluorescein angiography and B-scan ultrasonography, may be especially helpful in making the diagnosis of choroidal infiltration. Fluorescein angiography reveals diffuse pinpoint leakage, while B-scan ultrasonography

Unilateral Eye Findings: A Rare Herald of Acute Leukemia may reveal serous detachment or choroidal thickening $[22,23]$.

Intraocular manifestations of leukemia such as those described in these cases should prompt a thorough systemic workup. Systemic treatment is recommended first in these cases, but if not an option or if the ocular disease is not responsive, ocular radiation may be implemented. The dose of external beam radiation required in these cases is usually lower than that required for lymphoma and is most commonly 20 Gy delivered at fractions of 1.5-2 Gy daily [24]. If left untreated, leukemic ocular infiltrative disease may cause significant visual loss; however, it is usually very responsive to systemic treatment with chemotherapy, local radiation treatment to the eye, or a combination of both.

Physicians should be aware that unilateral eye findings can be an initial manifestation of recurrent leukemia, and in patients with a past history of leukemia, practitioners must maintain a heightened awareness for relapsed hematologic disease. Both of these cases show that leukemic involvement of the eye may mimic other disease, such as central serous retinopathy in the first case or infectious cellulitis in the second case, and the unilateral nature of symptoms may make the diagnosis of a systemic etiology particularly difficult. Prompt referral to an ophthalmologist will help determine if there is a high suspicion for relapsed leukemia and systemic treatment is often integral to resolution of the ocular problems.

\section{Statement of Ethics}

This study was conducted in compliance with the rules and regulations of the Health Insurance Portability and Accountability Act as well as in adherence to the Declaration of Helsinki and all other relevant federal and state laws.

\section{Disclosure Statement}

The authors have no financial disclosures or conflicts of interest to report.
Ocul Oncol Pathol 2016;2:166-170 DOI: $10.1159 / 000442951$ 


\section{References}

1 Kincaid MC, Green WR: Ocular and orbital involvement in leukemia. Surv Ophthalmol 1983;27:211-232.

2 Schachat AP: Ophthalmic manifestations of leukemia. Arch Ophthalmol 1989;107:697.

3 Ghosh K, Mitra S, Hiwase D: Choroidal infiltrates simulating fundal changes of acute leukemia during hematological recovery following high-dose chemotherapy in acute myelomonocytic leukemia in remission. Am J Hematol 2000;63:42-45.

4 Madjlessi F, Dann K, Althaus C, Sundmacher $\mathrm{R}$, Meckenstock G: Choroid infiltration in myelodysplastic syndrome. Klin Monbl Augenheilkd 1998;213:51-54.

5 Karataş M, Altan Yaycıoğlu R, Boğa C, Ulaş B: Akut myeloid lösemiye bağlı bilateral seröz maküla dekolmanı. Türk Oftalmol Derg 2014; 44:151-153.

6 Burns CA, Blodi FC, Williamson BK: Acute lymphocytic leukemia and central serous retinopathy. Trans Am Acad Ophthalmol Otolaryngol 1965;69:307-309.

7 Murphy JA, Pitts JF, Dudgeon J, Hogg RB, Harnett AN: Retinal detachments due to chronic lymphocytic leukaemia. Clin Lab Haematol 1991;13:217-220.

8 Hine JE, Kingham JD: Myelogenous leukemia and bilateral exudative retinal detachment. Ann Ophthalmol 1979;11:1867-1872.
9 Candoni A, Simeone E, Bandello F, Fanin R: Leukaemic infiltration of the retina at onset of Philadelphia-positive acute lymphoblastic leukaemia revealed by stratus optical coherence tomography. Br J Haematol 2006;133: 455.

10 Kim J, Chang W, Sagong M: Bilateral serous retinal detachment as a presenting sign of acute lymphoblastic leukemia. Korean J Ophthalmol 2010;24:245-248.

11 Yoshida A, Kawano Y-I, Eto T, et al: Serous retinal detachment in an elderly patient with Philadelphia-chromosome-positive acute lymphoblastic leukemia. Am J Ophthalmol 2005; 139:348-349.

12 Riss JM, Kaplanski G, Righini-Chossegros M, Harle JR, Escoffier P, Saracco JB: Bilateral serous detachment of neuroepithelium of the posterior pole disclosing acute leukemia. J Fr Ophtalmol 1990;13:563-568.

13 Caillaux V, Querques G, Ramahefasolo C, Darugar A, Souied EH: Bilateral macular serous retinal detachment revealing acute myeloblastic leukemia. Retin Cases Brief Rep 2013;7:62-66.

14 Fackler TK, Bearelly S, Odom T, Fekrat S, Cooney MJ: Acute lymphoblastic leukemia presenting as bilateral serous macular detachments. Retina 2006;26:710-712.

15 Wu L, Calderón M, Hernández G, Marbis J, Ramírez V: Bilateral exudative retinal detachment as the first sign of relapsing acute myelogenous leukaemia. Clin Experiment Ophthalmol 2006;34:623-625.
16 Chng WJ, Mow BM, Sundar G: Leukaemic infiltration of the choroid. Eur J Haematol 2005; 74:91.

17 Golan S, Goldstein M: Acute lymphocytic leukemia relapsing as bilateral serous retinal detachment: a case report. Eye (Lond) 2011;25: 1375-1378.

18 Tumuluri K, Woo T, Crowston J, Healey PR Gottlieb D, Maloof AJ: Bilateral leukemic orbital infiltration presenting as proptosis and narrow-angle glaucoma. Ophthal Plast $\mathrm{Re}$ constr Surg 2004;20:248-250.

19 Shields JA, Shields CL: Intraocular Tumors: An Atlas and Textbook. Philadelphia, Lippincott Williams \& Wilkins, 2008.

20 Allen RA, Straatsma BR: Ocular involvement in leukemia and allied disorders. Arch Ophthalmol 1961;66:490-508.

21 Stewart MW, Gitter KA, Cohen G: Acute leukemia presenting as a unilateral exudative retinal detachment. Retina 1989;9:110-114.

22 Miyamoto K: Serous retinal detachment caused by leukaemic choroidal infiltration during complete remission. Br J Ophthalmol 2000;84:1318a-1318.

23 Abramson DH, Jereb B, Wollner N, Murphy L, Ellsworth RM: Leukemic ophthalmopathy detected by ultrasound. J Pediatr Ophthalmol Strabismus 1983;20:92-97.

24 Brady LW, Shields JA, Augsburger JJ, Day JL: Malignant intraocular tumors. Cancer 1982; 49:578-585. 